\title{
SCHRÖDINGER OPERATOR ON THE AXIS WITH POTENTIALS DEPENDING ON TWO PARAMETERS
}

\author{
R. R. GADYL'SHIN AND I. KH. KHUSNULLIN
}

Dedicated to Vasilǐ Mikhaìlovich Babich, a remarkable mathematician and personality

\begin{abstract}
A Schrödinger operator on the axis is considered; its localized potential is the sum of a small potential and certain potentials with contracting supports, which can increase unboundedly when their supports are contracted. Sufficient conditions are presented for the absence (or existence) of eigenvalues for such an operator. In the case where eigenvalues exist, their asymptotic expansion is constructed.
\end{abstract}

\section{INTRODUCTION}

The study of one-dimensional Schrödinger operators with small potential has a long history. In [1, Chapter III, §22], it was proved that, in the case of a small rectangular potential well, the Schrödinger operator has a single eigenvalue, and the asymptotic expansion of this eigenvalue with respect to a small parameter was constructed.

The operator

$$
-\frac{d^{2}}{d x^{2}}+\delta W(x), \quad 0<\delta \ll 1,
$$

where $W(x)$ is an integrable real function that decays sufficiently rapidly at infinity, was studied in 2, 3, 4, 5.

By the Birman-Schwinger method, it was proved that if

$$
\langle W\rangle:=\int_{-\infty}^{\infty} W(x) d x>0,
$$

then the operator (0.1) has no eigenvalues; if

$$
\langle W\rangle \leq 0,
$$

then the operator has a single eigenvalue $\lambda_{\delta}$ with the asymptotic expansions

$$
\lambda_{\delta}=-\delta^{2} \frac{\langle W\rangle^{2}}{4}(1+\mathcal{O}(\delta)) \quad \text { if } \quad\langle W\rangle<0
$$

and

$$
\lambda_{\delta}=-\delta^{4} \frac{1}{4}\left(\int_{-\infty}^{\infty}\left(\int_{-\infty}^{x} W(t) d t\right)^{2} d x\right)^{2}(1+\mathcal{O}(\delta))
$$

in the critical case where $\langle W\rangle=0$.

2010 Mathematics Subject Classification. Primary 35J10, Secondary 35P20.

Key words and phrases. Schrödinger operator, perturbation.

Supported by RFBR-Volga region (grant no. 08-01-97016-r), a grant of the President of Russia for support of leading scientific schools (NSh-6249.2010.1) and by FPTs (02.740.110612). The second author was also supported by a grant of the President of Russia for support of young Doctors of Science (MD453.2010.1). 
Obviously, the operator

$$
-\frac{d^{2}}{d x^{2}}+\mu^{-1} h W(x)
$$

where $0<\mu, h$ and $\mu^{-1} h \ll 1$, reduces to the operator (0.1) by the substitution $\delta=$ $\mu^{-1} h$. Therefore, from (0.4) and (0.5) we see that if inequality (0.2) is fulfilled, then the operator (0.6) has no eigenvalues; if inequality (0.3) is fulfilled, then there exists a unique eigenvalue $\lambda_{\mu, h}$ with the asymptotic expansions

$$
\lambda_{\mu, h}=-\left(h \mu^{-1}\right)^{2} \frac{\langle W\rangle^{2}}{4}\left(1+\mathcal{O}\left(h \mu^{-1}\right)\right) \quad \text { if } \quad\langle W\rangle<0
$$

and

$$
\lambda_{\mu, h}=-\left(h \mu^{-1}\right)^{4} \frac{1}{4}\left(\int_{-\infty}^{\infty}\left(\int_{-\infty}^{x} W(t) d t\right)^{2} d x\right)^{2}\left(1+\mathcal{O}\left(h \mu^{-1}\right)\right)
$$

if $\langle W\rangle=0$.

Also, it is obvious that, by the change of variable $\xi=h^{-1} x$, the operator

$$
-\frac{d^{2}}{d x^{2}}+\mu^{-1} W\left(\frac{x}{h}\right)
$$

reduces to an operator of the form (0.1) for $\delta=h^{2} \mu^{-1}$. Therefore, for $h^{2} \mu^{-1} \ll 1$, formulas (0.4) and (0.5) show that if inequality (0.2) is fulfilled, then the operator (0.9) has no eigenvalues. If inequality (0.3) is fulfilled, then there exists a unique eigenvalue $\lambda_{\mu, h}$ with the asymptotic expansions

$$
\lambda_{\mu, h}=-\left(h \mu^{-1}\right)^{2} \frac{\langle W\rangle^{2}}{4}\left(1+\mathcal{O}\left(h^{2} \mu^{-1}\right)\right) \quad \text { if } \quad\langle W\rangle<0
$$

and

$$
\lambda_{\mu, h}=-h^{2}\left(h \mu^{-1}\right)^{4} \frac{1}{4}\left(\int_{-\infty}^{\infty}\left(\int_{-\infty}^{x} W(t) d t\right)^{2} d x\right)^{2}\left(1+\mathcal{O}\left(h^{2} \mu^{-1}\right)\right)
$$

if $\langle W\rangle=0$.

From (0.7) and (0.10) it follows that if $\langle W\rangle<0$, then the leading terms of the asymptotic expansions for the eigenvalues of the operators (0.6) and (0.9) coincide. In the critical case where $\langle W\rangle=0$, from (0.8) and (0.11) it follows that the leading terms of the asymptotic expansions for the eigenvalues of these operators have distinct orders of smallness.

In the present paper, we study the eigenvalues of the operator

$$
\mathcal{H}^{\mu, h}:=-\frac{d^{2}}{d x^{2}}+\mu^{-1}\left(\sum_{j=1}^{n} V_{j}\left(\frac{x-x_{j}}{h}\right)+h W(x)\right), \quad 0<h \ll 1,
$$

under the assumption that there exists $\gamma>0$ such that

$$
\mu^{-1} h^{1 / 2}=o\left(h^{\gamma}\right)
$$

Here, the $x_{j}$ are arbitrary distinct numbers, and $V_{1}(x), \ldots, V_{n}(x), W(x)$ are complexvalued functions of class $C_{0}^{\infty}(\mathbb{R})$ at least two of which are nonzero. Clearly, even for real functions, the operator $\mathcal{H}^{\mu, h}$ cannot be reduced to (0.1) by a change of variables.

The operator $\mathcal{H}_{0}:=-\frac{d^{2}}{d x^{2}}$ in $L_{2}(\mathbb{R})$ with domain $W_{2}^{2}(\mathbb{R})$ is selfadjoint, its discrete spectrum is empty, and the essential spectrum coincides with the nonnegative real axis. Since the functions $V_{j}$ and $W$ have compact support, the essential spectrum of the operator $\mathcal{H}^{\mu, h}$ regarded as an operator in $L_{2}(\mathbb{R})$ with domain $W_{2}^{2}(\mathbb{R})$ coincides with the nonnegative real axis (for the case of complex functions, see, e.g., [6]). 


\section{$\S 1$. Statement of main Results}

Denote

$$
\varkappa \mathbf{V}, W:=\sum_{j=1}^{n}\left\langle V_{j}\right\rangle+\langle W\rangle .
$$

In the present paper, we prove the following statements.

Theorem 1.1. Under condition (0.12), if $\operatorname{Re} \varkappa_{\mathbf{V}, W}>0$, then the operator $\mathcal{H}^{\mu, h}$ has no eigenvalues converging to zero.

If $\operatorname{Re} \varkappa_{\mathbf{V}, W}<0$, then the operator $\mathcal{H}^{\mu, h}$ has a unique eigenvalue $\lambda^{\mu, h}$ converging to zero; this eigenvalue is simple and

$$
\lambda^{\mu, h}=-\left(\mu^{-1} h\right)^{2} \frac{\varkappa_{\mathbf{V}, W}^{2}}{4}\left(1+\mathcal{O}\left(\mu^{-1} h\right)\right) .
$$

Formula (1.1) is a natural generalization of identities (0.7) and (0.10) to complexvalued potentials.

In the critical case, for simplicity of the statements and calculations below, we only deal with real-valued functions $V_{1}(x), \ldots, V_{n}(x), W(x)$.

Theorem 1.2. Let conditions (0.12) and $\langle W\rangle=\left\langle V_{j}\right\rangle=0, j=1, \ldots, n$, be fulfilled. Then the operator $\mathcal{H}^{\mu, h}$ has a unique eigenvalue $\lambda^{\mu, h}$ converging to zero. This eigenvalue is simple and has the asymptotic expansion

$$
\lambda^{\mu, h}=-\frac{1}{4}\left(\mu^{-1} h\right)^{4}\left(\int_{-\infty}^{\infty}\left(\int_{-\infty}^{x} W(t) d t\right)^{2} d x\right)^{2}\left(1+\mathcal{O}\left(h+\mu^{-1} h\right)\right) .
$$

This theorem shows that, in the version $\varkappa_{\mathbf{V}, W}=0$ of the critical case in question, the potential $\mu^{-1} h W(x)$ is "principal", and the leading term of the asymptotic expansion of the eigenvalue (1.2) coincides with the leading term of the asymptotic expansion of the eigenvalue (0.8) for the operator (0.6).

For clarity, the remaining versions of the critical case where $\varkappa_{\mathbf{V}, W}=0$ are considered for two potentials.

Theorem 1.3. Assume condition (0.12). Let

$$
\mathcal{H}^{\mu, h}=-\frac{d^{2}}{d x^{2}}+\mu^{-1}\left(V\left(\frac{x}{h}\right)+h W(x)\right),
$$

and let $\langle V\rangle+\langle W\rangle=0$, but $\langle W\rangle\langle V\rangle \neq 0$. Then the operator $\mathcal{H}^{\mu, h}$ has a unique eigenvalue $\lambda^{\mu, h}$ converging to zero. This eigenvalue is simple and has the asymptotic expansion

$$
\begin{aligned}
\lambda^{\mu, h}=- & \frac{1}{4}\left(\mu^{-1} h\right)^{4}\left(\int_{\operatorname{supp} W}\left(\int_{p}^{x} W(t) d t\right)^{2} d x+q\langle W\rangle^{2}\right)^{2} \\
& \times\left(1+\mathcal{O}\left(h+\mu^{-1} h\right)\right) \quad \text { if } 0 \notin \operatorname{supp} W,
\end{aligned}
$$

where $q=\operatorname{dist}\{0, \operatorname{supp} W\}, p=\max _{x \in \operatorname{supp} W}|x|$, and

$$
\begin{aligned}
\lambda^{\mu, h}=- & \frac{1}{4}\left(\mu^{-1} h\right)^{4}\left(\int_{-\infty}^{0}\left(\int_{-\infty}^{x} W(t) d t\right)^{2} d x+\int_{0}^{\infty}\left(\int_{x}^{\infty} W(t) d t\right)^{2} d x\right) \\
& \times\left(1+\mathcal{O}\left(h+\mu^{-1} h\right)\right) \text { if } 0 \in \operatorname{supp} W .
\end{aligned}
$$

Comparing (1.3) and (1.4) with (0.8) and (0.11), we see that, in the case in question, the contribution of the narrow potential $\mu^{-1} V\left(\frac{x}{h}\right)$ to the leading term of the asymptotic expansion in question is as significant as that of the potential $\mu^{-1} h W(x)$. 
Theorem 1.4. Under condition (0.12), let

$$
\mathcal{H}^{\mu, h}=-\frac{d^{2}}{d x^{2}}+\mu^{-1}\left(V\left(\frac{x-x_{1}}{h}\right)-V\left(\frac{x-x_{2}}{h}\right)\right), \quad x_{1}<x_{2},
$$

and suppose $\langle V\rangle \neq 0$. Then the operator $\mathcal{H}^{\mu, h}$ has a unique eigenvalue $\lambda^{\mu, h}$ converging to zero. This eigenvalue is simple and has the asymptotic expansion

$$
\lambda^{\mu, h}=-\frac{1}{4}\left(\mu^{-1} h\right)^{4}\left(\int_{x_{1}}^{x_{2}}\left(\int_{-\infty}^{x} V(t) d t\right)^{2} d x\right)^{2}\left(1+\mathcal{O}\left(h+\mu^{-1} h\right)\right) .
$$

Formula (1.5) shows that, in this situation, the smallness order of the leading term of the eigenvalue differs from the case of a single narrow potential (see (0.11) ) and is equal to that order in the case of a single small potential (see (0.8)).

\section{§2. Preliminary information}

In [7, the operator

$$
\mathcal{H}_{\varepsilon}=-\frac{d^{2}}{d x^{2}}+\varepsilon \mathcal{L}_{\varepsilon}
$$

was considered, where $0<\varepsilon \ll 1$ is a small parameter and $\mathcal{L}_{\varepsilon}$ is an arbitrary localized second order linear operator. Namely, there exists a bounded domain $Q \subset \mathbb{R}$ such that

$$
\mathcal{L}_{\varepsilon}: W_{2, \text { loc }}^{2}(\mathbb{R}) \rightarrow L_{2}(\mathbb{R} ; Q),
$$

where

$$
L_{2}(\mathbb{R} ; Q):=\left\{u: u \in L_{2}(\mathbb{R}), \operatorname{supp} u \subset \bar{Q}\right\}
$$

and

$$
\left\|\mathcal{L}_{\varepsilon} u\right\|_{L_{2}(\mathbb{R})} \leq C_{1}\|u\|_{W_{2}^{2}(Q)},
$$

where the constant $C_{1}$ does not depend on $\varepsilon$. In [6], it was proved that the operator $\mathcal{H}_{\varepsilon}$ in $L_{2}(\mathbb{R})$ with domain $W_{2}^{2}(\mathbb{R})$ is closed and its essential spectrum coincides with the nonnegative real semiaxis.

For small complex $k$, we define an operator $A(k): L_{2}(\mathbb{R} ; Q) \rightarrow W_{2, \text { loc }}^{2}(\mathbb{R})$ as follows:

$$
A(k) g:=\frac{1}{2 k} \int_{-\infty}^{\infty} e^{-k|x-t|} g(t) d t .
$$

The definition of $A(k)$ implies that if $\mathcal{R}(\lambda)$ is the resolvent of the operator $-\frac{d^{2}}{d x^{2}}$ in $L_{2}(\mathbb{R})$, then $A(k)=\mathcal{R}\left(-k^{2}\right)$ on functions in $L_{2}(\mathbb{R} ; Q)$ for $\operatorname{Re} k>0$. We denote by $I$ the identity operator and by

$$
\widetilde{A}(k): L_{2}(\mathbb{R} ; Q) \rightarrow W_{2}^{2}(Q) \quad \text { and } \quad T_{\varepsilon}(k): L_{2}(\mathbb{R} ; Q) \rightarrow L_{2}(\mathbb{R} ; Q)
$$

the operators acting as follows:

$$
\begin{aligned}
\widetilde{A}(k) g & :=A(k) g-\frac{1}{2 k}\langle g\rangle, \\
T_{\varepsilon}(k) g & :=\mathcal{L}_{\varepsilon} \widetilde{A}(k) g .
\end{aligned}
$$

In [7, the following results were obtained.

Proposition 1. For small $k$, the equation

$$
2 k+\varepsilon \mathcal{F}_{\varepsilon}(k)=0,
$$

where

has a unique solution $k_{\varepsilon}$.

$$
\mathcal{F}_{\varepsilon}(k)=\left\langle\left(I+\varepsilon T_{\varepsilon}(k)\right)^{-1} \mathcal{L}_{\varepsilon(\mu, h)}[1]\right\rangle,
$$


If $\operatorname{Re} k_{\varepsilon}<0$, then the operator $\mathcal{H}_{\varepsilon}$ has no eigenvalues converging to zero.

If $\operatorname{Re} k_{\varepsilon}>0$, then the operator $\mathcal{H}_{\varepsilon}$ has a unique eigenvalue $\lambda_{\varepsilon}$ converging to zero, and

$$
\lambda_{\varepsilon}=-k_{\varepsilon}^{2} .
$$

The definition of the function $\mathcal{F}_{\varepsilon}(k)$ shows that, for every $N \geq 2$, we have

$$
\mathcal{F}_{\varepsilon}(k)=\left\langle\mathcal{L}_{\varepsilon}[1]\right\rangle+\sum_{j=1}^{N-1}(-1)^{j} \varepsilon^{j}\left\langle T_{\varepsilon}^{j}(k) \mathcal{L}_{\varepsilon}[1]\right\rangle+\varepsilon^{N} \mathcal{F}_{\varepsilon, N}(k),
$$

where $\mathcal{F}_{\varepsilon, N}(k)$ is a function holomorphic in $k$ and uniformly bounded with respect to $\varepsilon$.

\section{§3. Reduction of the operator $\mathcal{H}^{\mu, h}$ to the operator $\mathcal{H}_{\varepsilon}$}

Lemma 3.1. Suppose $-\infty<a<x_{0}<b<\infty$ and $c_{0}>0$. Then

$$
\int_{x_{0}-c_{0} h}^{x_{0}+c_{0} h}|u|^{2} \mathrm{~d} x \leq C_{2} h\|u\|_{W_{2}^{2}(a, b)}^{2}
$$

for all functions $u$ of class $W_{2}^{2}(a, b)$, where the constant $C_{2}$ is independent of $h$.

Proof. Without loss of generality, we may assume that $[a, b] \subset(0,1)$. We denote by $\stackrel{\circ}{W}_{2}^{1}(0,1)$ the completion of $C_{0}^{\infty}(0,1)$ with respect to the norm $W_{2}^{1}(0,1)$. In [8], it was proved that for all functions $v \in \dot{W}_{2}^{1}(0,1)$ we have

$$
\int_{x_{0}-h c_{0}}^{x_{0}+h c_{0}}|v(x)|^{2} \mathrm{~d} x \leq C_{3} h\|v\|_{W_{2}^{1}(0,1)}^{2},
$$

where the constant $C_{3}$ does not depend on $h$ and $v$.

It is well known (see, e.g., 9]) that there exists a bounded linear extension operator $P: W_{2}^{1}(a, b) \rightarrow \stackrel{\circ}{W}_{2}^{1}(0,1)$ such that $u=P u$ for $x \in(a, b)$ for every $u \in W_{2}^{1}(a, b)$. Therefore, by (3.1), we have

$$
\begin{aligned}
\int_{x_{0}-h c_{0}}^{x_{0}+h c_{0}}|u(x)|^{2} \mathrm{~d} x & =\int_{x_{0}-h c_{0}}^{x_{0}+h c_{0}}|P u(x)|^{2} \mathrm{~d} x \leq C_{3} h\|P u\|_{W_{2}^{1}(0,1)}^{2} \leq C_{3}\|P\|^{2} h\|u\|_{W_{2}^{1}(a, b)}^{2} \\
& =C_{2} h\|u\|_{W_{2}^{1}(a, b)}^{2} \leq C_{2} h\|u\|_{W_{2}^{2}(a, b)}^{2}
\end{aligned}
$$

for every $u \in W_{2}^{1}(a, b)$. The lemma is proved.

By $\mathcal{V}_{h}$, we denote the operator of multiplication by the function

$$
\sum_{j=1}^{n} V_{j}\left(\frac{x-x_{j}}{h}\right)+h W(x) .
$$

Lemma 3.2. Let $Q$ be an arbitrary finite interval such that

$$
\operatorname{supp} W \cup \bigcup_{j=1}^{n}\left\{x_{j}\right\} \subset Q .
$$

Then, for every $u \in W_{2, \text { loc }}^{2}(\mathbb{R})$ and all sufficiently small $h$, we have the estimate

$$
\left\|h^{-\frac{1}{2}} \mathcal{V}_{h} u\right\|_{L_{2}(\mathbb{R})} \leq C_{4}\|u\|_{W_{2}^{2}(Q)},
$$

where $C_{4}$ is a constant independent of $h$. 
Proof. Let $\mathcal{V}_{h}^{(j)}$ and $\mathcal{W}$ denote the operators of multiplication by the functions $V_{j}\left(\frac{x-x_{j}}{h}\right)$ and $W(x)$, respectively. Obviously,

$$
\left\|\mathcal{V}_{h} u\right\|_{L_{2}(\mathbb{R})} \leq \sum_{j=1}^{n}\left\|\mathcal{V}_{h}^{(j)} u\right\|_{L_{2}(\mathbb{R})}+h\|\mathcal{W} u\|_{L_{2}(\mathbb{R})} .
$$

Let $c>0$ be a number such that $\operatorname{supp} V_{j}(t) \subset(-c, c)$ for every $j$. Then, by Lemma 3.1,

$$
\begin{aligned}
\left\|\mathcal{V}_{h}^{(j)} u\right\|_{L_{2}(\mathbb{R})}^{2} & =\int_{x_{j}-h c}^{x_{j}+h c}\left|V_{j}\left(\frac{x-x_{j}}{h}\right) u(x)\right|^{2} \mathrm{~d} x \\
& \leq \max _{t \in[-c, c]}\left|V_{j}(t)\right|^{2} \int_{x_{j}-h c}^{x_{j}+h c}|u(x)|^{2} \mathrm{~d} x \leq \widetilde{C}_{j} h\|u\|_{W_{2}^{2}(Q)}^{2} .
\end{aligned}
$$

It is obvious that

$$
\|\mathcal{W} u\|_{L_{2}(\mathbb{R})}=\|\mathcal{W} u\|_{L_{2}(Q)} \leq \widetilde{C}_{W}\|u\|_{L_{2}(Q)} \leq \widetilde{C}_{W}\|u\|_{W_{2}^{2}(Q)} .
$$

Relations (3.4)-(3.6) lead to estimate (3.3).

Lemma 3.3. Suppose

$$
0<h \ll 1, \quad h=o\left(\mu^{2}\right),
$$

and let $Q$ satisfy condition (3.2),

$$
\varepsilon(\mu, h):=\mu^{-1} h^{\frac{1}{2}}, \quad \mathcal{L}_{\varepsilon(\mu, h)}:=h^{-\frac{1}{2}} \mathcal{V}_{h}
$$

Then

$$
0<\varepsilon(\mu, h) \ll 1,
$$

the operator $\mathcal{H}^{\mu, h}$ can be represented in the form (2.1), and relations (2.2) and (2.3) are valid.

Proof. Inequality (3.9) follows from (3.7) and (3.8). By (3.8), we have

$$
\mathcal{H}^{\mu, h}=-\frac{d^{2}}{d x^{2}}+\mu^{-1} \mathcal{V}_{h}=-\frac{d^{2}}{d x^{2}}+\mu^{-1} h^{\frac{1}{2}} h^{-\frac{1}{2}} \mathcal{V}_{h}=-\frac{d^{2}}{d x^{2}}+\varepsilon(\mu, h) \mathcal{L}_{\varepsilon(\mu, h)} .
$$

Consequently, the operator $\mathcal{H}^{\mu, h}$ can be represented in the form (2.1). From (3.8) and (3.3), it follows that

$$
\left\|\mathcal{L}_{\varepsilon(\mu, h)} u\right\|_{L_{2}(\mathbb{R})}=\left\|h^{-\frac{1}{2}} \mathcal{V}_{h} u\right\|_{L_{2}(\mathbb{R})} \leq C_{5}\|u\|_{W_{2}^{2}(Q)} .
$$

In other words, inequality (2.3) is valid for the operator $\mathcal{L}_{\varepsilon(\mu, h)}$. Now, obviously, the definition of $\mathcal{L}_{\varepsilon(\mu, h)}$ implies relation (2.2).

In the sequel, we assume that $Q$ is an arbitrary finite interval satisfying (3.2).

\section{§4. Proof of Theorem 1.1}

Using (2.5), (2.8), and Lemma 3.3 we get

$$
\begin{aligned}
\mathcal{F}_{\varepsilon(\mu, h)}(k)=h^{-\frac{1}{2}} & \left(\left\langle\mathcal{V}_{h}[1]\right\rangle+\sum_{j=1}^{N-1}(-1)^{j} \mu^{-j}\left\langle\left(\mathcal{V}_{h} \widetilde{A}(k)\right)^{j}\left(\mathcal{V}_{h}[1]\right)\right\rangle\right) \\
& +\left(\mu^{-1} h^{\frac{1}{2}}\right)^{N} \mathcal{F}_{\varepsilon(\mu, h), N}(k) .
\end{aligned}
$$

We prove that the following estimate uniform in $k$ is valid for small $k$ :

$$
\left|\left\langle\left(\mathcal{V}_{h} \widetilde{A}(k)\right)^{j} \mathcal{V}_{h}[1]\right\rangle\right|=\mathcal{O}\left(h^{j+1}\right), \quad j \geq 1 .
$$


The definition (2.4) of $\widetilde{A}(k)$ shows that

$$
\widetilde{A}(k) g=\frac{1}{2 k} \int_{-\infty}^{\infty}\left(e^{-k|x-y|}-1\right) g(y) d y
$$

for $g \in L_{2}(\mathbb{R} ; Q)$. Expanding the function $e^{-k|x-y|}$ in a series in $k$, we obtain

$$
\widetilde{A}(k) g=\frac{1}{2} \sum_{i=1}^{\infty}(-1)^{i} \frac{k^{i-1}}{i !} \int_{-\infty}^{\infty}|x-y|^{i} g(y) d y, \quad x \in Q .
$$

Consequently,

$$
\left(\mathcal{V}_{h} \tilde{A}(k)\right) \mathcal{V}_{h}[1]=\frac{1}{2} h \mathcal{V}_{h} F_{1},
$$

where

$$
\begin{aligned}
F_{1}(x, h, k) & =\sum_{i=1}^{\infty}(-1)^{i} \frac{k^{i-1}}{i !} f_{1, i}(x, h), \\
f_{1, i}(x, h) & =\int_{-\infty}^{\infty}\left(\sum_{j=1}^{n}\left|x-x_{j}-h y\right|^{i} V_{j}(y)+|x-y|^{i} W(y)\right) d y .
\end{aligned}
$$

From the definition of $f_{1, i}$, it follows that for sufficiently small $h$ we have

$$
\left|f_{1, i}(x, h)\right| \leq C_{5} M^{i}, \quad x \in \bar{Q},
$$

where $M=2 R$ and $R$ is a positive number such that the supports of the functions $V_{j}(x)$ and $W(x)$ and the points $x_{j}$ lie in $(-R, R)$. Consequently, the series (4.5) converges uniformly for $x \in Q$ and small $k$ and $h$, and

$$
\left|\mathcal{V}_{h} F_{1}(x, h, k)\right| \leq C_{6} .
$$

Therefore, by (4.4) and (4.5),

$$
\left\langle\left(\mathcal{V}_{h} \widetilde{A}(k)\right) \mathcal{V}_{h}[1]\right\rangle=\frac{1}{2} h \sum_{i=1}^{\infty}(-1)^{i} \frac{k^{i-1}}{i !}\left\langle\mathcal{V}_{h} f_{1, i}(x, h)\right\rangle .
$$

Hence, by (4.6), we obtain

$$
\begin{aligned}
\left|\left\langle\left(\mathcal{V}_{h} \widetilde{A}(k)\right) \mathcal{V}_{h}[1]\right\rangle\right| & \leq \frac{1}{2} h \sum_{i=1}^{\infty} \frac{|k|^{i-1}}{i !}\left|\left\langle\mathcal{V}_{h} f_{1, i}(x, h)\right\rangle\right| \\
& \leq \frac{1}{2} h \sum_{i=1}^{\infty} \frac{|k|^{i-1}}{i !}\left\langle\left|\mathcal{V}_{h}[1]\right|\left|f_{1, i}(x, h)\right|\right\rangle \leq h C_{6}\left\langle\left|\mathcal{V}_{h}[1]\right|\right\rangle
\end{aligned}
$$

Next,

$$
\begin{aligned}
\left\langle\left|\mathcal{V}_{h}[1]\right|\right\rangle & =\int_{-\infty}^{\infty}\left|\sum_{j=1}^{n} V_{j}\left(\frac{y-x_{j}}{h}\right)+h W(y)\right| d y \\
& \leq h \sum_{j=1}^{n} \int_{-\infty}^{\infty}\left|V_{j}(y)\right| d y+h \int_{-\infty}^{\infty}|W(y)| d y \leq C_{7} h
\end{aligned}
$$

The last two inequalities combine to yield

$$
\left|\left\langle\left(\mathcal{V}_{h} \tilde{A}(k)\right) \mathcal{V}_{h}[1]\right\rangle\right| \leq C_{7} h^{2} .
$$

We have proved (4.2) for $j=1$.

For $j=2$, we use the representation

$$
\left(\mathcal{V}_{h} \widetilde{A}(k)\right)^{2} \mathcal{V}_{h}[1]=\frac{1}{2} h \mathcal{V}_{h} \widetilde{A}(k) \mathcal{V}_{h} F_{1}=\frac{1}{4} h^{2} \mathcal{V}_{h} F_{2},
$$


where

$$
\begin{aligned}
F_{2}(x, h, k) & =\sum_{i=1}^{\infty}(-1)^{i} \frac{k^{i-1}}{i !} f_{2, i}(x, h, k), \\
f_{2, i}(x, h, k) & =\int_{-\infty}^{\infty}\left(\sum_{j=1}^{n}\left|x-x_{j}-h y\right|^{i} V_{j}(y) F_{1}\left(x_{j}+h y, h, k\right)+|x-y|^{i} W(y) F_{1}(y, h, k)\right) d y .
\end{aligned}
$$

Taking (4.7) into account and repeating the above estimates, we see that (4.2) is true for $j=2$.

The further proof of (4.2) proceeds by an easy induction.

Using (4.1), (3.8), (4.2), (0.12), and the fact that $N$ is arbitrary, we get

$$
\varepsilon(\mu, h) \mathcal{F}_{\varepsilon(\mu, h)}(k)=\mu^{-1}\left\langle\mathcal{V}_{h}[1]\right\rangle+\mu^{-2} h^{2} \mathcal{G}(k, h, \mu),
$$

where $\mathcal{G}(k, h, \mu)$ is a function holomorphic in $k$ and uniformly bounded in $\mu$ and $h$.

By the definition of $\mathcal{V}_{h}$,

$$
\begin{aligned}
\left\langle\mathcal{V}_{h}[1]\right\rangle & =\int_{-\infty}^{\infty}\left(\sum_{j=1}^{n} V_{j}\left(\frac{x-x_{j}}{h}\right)+h W(x)\right) d x \\
& =h \sum_{j=1}^{n} \int_{-\infty}^{\infty} V_{j}(t) d t+h \int_{-\infty}^{\infty} W(x) d x=h \varkappa_{\mathbf{V}, W} .
\end{aligned}
$$

Relations (4.9) and (4.10) show that equation (2.6) takes the form

$$
k=-\frac{h}{2 \mu}\left(\varkappa_{\mathbf{V}, W}+\mu^{-1} h \mathcal{G}(k, h, \mu)\right) .
$$

Combining this with Proposition 1, we obtain Theorem 1.1

\section{§5. Proof of Theorems 1.21 .4}

First, we derive a general formula for the eigenfrequency in the critical case where

$$
\varkappa_{\mathbf{V}, W}=\left\langle\mathcal{V}_{h}[1]\right\rangle=0 \text {. }
$$

(By an eigenfrequency, we mean a solution of equation (2.6), in the notation (3.8).)

From (4.1), (5.1), (4.2), (3.8), (0.12), and the fact that $N$ is arbitrary, it follows that

$$
\varepsilon(\mu, h) \mathcal{F}_{\varepsilon(\mu, h)}(k)=-\mu^{-2}\left\langle\left(\mathcal{V}_{h} \widetilde{A}(k)\right)\left(\mathcal{V}_{h}[1]\right)\right\rangle+\mu^{-3} h^{3} \mathcal{G}(k, h, \mu),
$$

where $\mathcal{G}(k, h, \mu)$ is a function holomorphic in $k$ and uniformly bounded in $\mu$ and $h$.

First, we prove that

$$
\left\langle\left(\mathcal{V}_{h} \tilde{A}(k)\right)\left(\mathcal{V}_{h}[1]\right)\right\rangle=-\frac{1}{2} \int_{-\infty}^{\infty} \mathcal{V}_{h}\left(\int_{-\infty}^{\infty}|x-y| \mathcal{V}_{h}[1](y) d y\right) d x+h^{2} k \mathcal{G}_{1}(k, h),
$$

where $\mathcal{G}_{1}(k, h)$ is a function holomorphic in $k$ and uniformly bounded in $h$. Using (4.3), we immediately obtain the equation

$$
\left\langle\left(\mathcal{V}_{h} \widetilde{A}(k)\right)\left(\mathcal{V}_{h}[1]\right)\right\rangle=-\frac{1}{2} \int_{-\infty}^{\infty} \mathcal{V}_{h}\left(\int_{-\infty}^{\infty}|x-y| \mathcal{V}_{h}[1](y) d y\right) d x+k \widetilde{\mathcal{G}}_{1}(k, h),
$$

where $\widetilde{\mathcal{G}}_{1}(k, h)$ is a function holomorphic in $k$ and uniformly bounded in $h$ that is defined by the equation

$$
\widetilde{\mathcal{G}}_{1}(k, h)=\frac{1}{2} \sum_{i=2}^{\infty}(-1)^{i} \frac{k^{i-2}}{i !} \int_{-\infty}^{\infty} \mathcal{V}_{h}\left(\int_{-\infty}^{\infty}|x-y|^{i} \mathcal{V}_{h}[1](y) d y\right) d x
$$


Similarly to (4.8), it can easily be proved that

$$
\left|\widetilde{\mathcal{G}}_{1}(k, h)\right| \leq C_{8} h^{2} .
$$

Now, (5.4) and (5.5) lead to (5.3).

We prove that, in the critical case (5.1) in question, the following identity is valid:

$$
\int_{-\infty}^{\infty} \mathcal{V}_{h}\left(\int_{-\infty}^{\infty}|x-y| \mathcal{V}_{h}[1](y) d y\right) d x=-2 \int_{-\infty}^{\infty}\left(\int_{-\infty}^{x} \mathcal{V}_{h}[1](t) d t\right)^{2} d x
$$

Indeed,

$$
\begin{aligned}
\int_{-\infty}^{\infty} & \mathcal{V}_{h}\left(\int_{-\infty}^{\infty}|x-y| \mathcal{V}_{h}[1](y) d y\right) d x \\
& =\int_{-\infty}^{\infty} \mathcal{V}_{h}\left(\int_{-\infty}^{x}(x-y) \mathcal{V}_{h}[1](y) d y\right) d x+\int_{-\infty}^{\infty} \mathcal{V}_{h}\left(\int_{x}^{\infty}(y-x) \mathcal{V}_{h}[1](y) d y\right) d x
\end{aligned}
$$

We change the order of integration in the second integral on the right:

$$
\int_{-\infty}^{\infty} \mathcal{V}_{h}\left(\int_{x}^{\infty}(y-x) \mathcal{V}_{h}[1](y) d y\right) d x=\int_{-\infty}^{\infty} \mathcal{V}_{h}\left(\int_{-\infty}^{y}(y-x) \mathcal{V}_{h}[1](x) d x\right) d y
$$

The last two identities imply

$$
\int_{-\infty}^{\infty} \mathcal{V}_{h}\left(\int_{-\infty}^{\infty}|x-y| \mathcal{V}_{h}[1](y) d y\right) d x=2 \int_{-\infty}^{\infty} \mathcal{V}_{h}\left(\int_{-\infty}^{x}(x-y) \mathcal{V}_{h}[1](y) d y\right) d x
$$

Furthermore, integration by parts yields

$$
\int_{-\infty}^{x}(x-y) \mathcal{V}_{h}[1](y) d y=\int_{-\infty}^{x} \int_{-\infty}^{y} \mathcal{V}_{h}[1](t) d t d y
$$

From the last two equations, we obtain

$$
\int_{-\infty}^{\infty} \mathcal{V}_{h}\left(\int_{-\infty}^{\infty}|x-y| \mathcal{V}_{h}[1](y) d y\right) d x=2 \int_{-\infty}^{\infty} U^{\prime}(x) \int_{-\infty}^{x} U(y) d y d x
$$

where

$$
U(z)=\int_{-\infty}^{z} \mathcal{V}_{h}[1](t) d t .
$$

Integrating the right-hand side of identity (5.7) by parts and taking into account the fact that $\left\langle\mathcal{V}_{h}[1]\right\rangle=0$, we obtain

$$
\int_{-\infty}^{\infty} \mathcal{V}_{h}\left(\int_{-\infty}^{x} \int_{-\infty}^{y} \mathcal{V}_{h}[1](t) d t d y\right)(x) d x=-\int_{-\infty}^{\infty}\left(\int_{-\infty}^{x} \mathcal{V}_{h}[1](t) d t\right)^{2} d x
$$

The last two identities result in (5.6).

By (5.2), (5.3), and (5.6), we have

$$
\begin{aligned}
\varepsilon(\mu, h) \mathcal{F}_{\varepsilon(\mu, h)}(k)= & -\mu^{-2} \int_{-\infty}^{\infty}\left(\int_{-\infty}^{x} \mathcal{V}_{h}[1](t) d t\right)^{2} d x \\
& -\mu^{-2} h^{2} k \mathcal{G}_{1}(k, h)+\mu^{-3} h^{3} \mathcal{G}(k, h, \mu) .
\end{aligned}
$$

Using the definition of $\mathcal{V}_{h}$ and the fact that

$$
\int_{-\infty}^{x} V_{j}\left(\frac{t-x_{j}}{h}\right) d t=h \int_{-\infty}^{\left(x-x_{j}\right) h^{-1}} V_{j}(t) d t
$$

we represent (5.9) in the form

$$
\varepsilon(\mu, h) \mathcal{F}_{\varepsilon(\mu, h)}(k)=-\mu^{-2} h^{2}\left(\tilde{\varkappa}_{\mathbf{V}, W}+k \mathcal{G}_{1}(k, h)-\mu^{-1} h \mathcal{G}(k, h, \mu)\right),
$$


where

$$
\tilde{\varkappa}_{\mathbf{V}, W}:=\int_{-\infty}^{\infty}\left(\sum_{j=1}^{n} \int_{-\infty}^{\left(x-x_{j}\right) h^{-1}} V_{j}(t) d t+\int_{-\infty}^{x} W(t) d t\right)^{2} d x .
$$

From (5.10), it follows that (2.6) takes the form

$$
k=\frac{h^{2}}{2 \mu^{2}}\left(\tilde{\varkappa}_{\mathbf{V}, W}+k \mathcal{G}_{1}(k, h)-\mu^{-1} h \mathcal{G}(k, h, \mu)\right) .
$$

Hence,

$$
k_{\varepsilon(\mu, h)}=\frac{h^{2}}{2 \mu^{2}} \tilde{\varkappa}_{\mathrm{V}, W}+\mathcal{O}\left(\mu^{-3} h^{3}\right) .
$$

Proof of Theorem 1.2. In the case in question, we have

$$
\langle W\rangle=0, \quad\left\langle V_{j}\right\rangle=0, \quad j=1, \ldots, n .
$$

Obviously, that there exist positive numbers $c_{j}$ such that $\operatorname{supp} V_{j}(x) \subset\left(-c_{j}, c_{j}\right)$. Therefore, for small $h$ we have

$$
\begin{aligned}
& \int_{-\infty}^{\infty}\left(\int_{-\infty}^{\left(x-x_{j}\right) h^{-1}} V_{j}(t) d t\right)^{2} d x=\int_{x_{j}-h c_{j}}^{x_{j}+h c_{j}}\left(\int_{-\infty}^{\left(x-x_{j}\right) h^{-1}} V_{j}(t) d t\right)^{2} d x=\mathcal{O}(h), \\
& \int_{-\infty}^{\infty} \int_{-\infty}^{\left(x-x_{j}\right) h^{-1}} V_{j}(t) d t \int_{p}^{x} W(t) d t d x=\mathcal{O}(h), \\
& \int_{-\infty}^{\infty} \int_{-\infty}^{\left(x-x_{i}\right) h^{-1}} V_{i}(t) d t \int_{-\infty}^{\left(x-x_{j}\right) h^{-1}} V_{j}(t) d t d x=0, \quad i \neq j .
\end{aligned}
$$

By (5.11) - (5.13), it follows that

$$
k_{\varepsilon}=\frac{1}{2} \mu^{-2} h^{2}\left(\int_{-\infty}^{\infty}\left(\int_{-\infty}^{x} W(t) d t\right)^{2} d x\right)+\mathcal{O}\left(\mu^{-2} h^{3}+\mu^{-3} h^{3}\right),
$$

and it remains to refer to Proposition 1. Theorem 1.2 is proved.

Proof of Theorem 1.3. In the case in question, we have

$$
\mathcal{V}_{h}[1]=V\left(\frac{x}{h}\right)+h W(x), \quad \operatorname{supp} V \subset\left(-c_{0}, c_{0}\right), \quad \operatorname{supp} W=\left[p_{1}, p_{2}\right],
$$

and formula (5.11) takes the form

$$
\tilde{\varkappa}_{\mathbf{V}, W}=\int_{-\infty}^{\infty}\left(\int_{-\infty}^{x h^{-1}} V(t) d t+\int_{-\infty}^{x} W(t) d t\right)^{2} d x .
$$

First, we consider the case where $p_{2}<0$. Then

$$
\begin{aligned}
\int_{-\infty}^{\infty} & \left(\int_{-\infty}^{x h^{-1}} V(t) d t+\int_{-\infty}^{x} W(t) d t\right)^{2} d x \\
& =\int_{p_{1}}^{p_{2}}\left(\int_{p_{1}}^{x} W(t) d t\right)^{2} d x-\left(p_{2}+h c_{0}\right)\langle W\rangle^{2}+\int_{-c_{0} h}^{c_{0} h}\left(\int_{-\infty}^{x h^{-1}} V(t) d t+\langle W\rangle\right)^{2} d x \\
& =\int_{\text {supp } W}\left(\int_{p}^{x} W(t) d t\right)^{2} d x+q\langle W\rangle^{2}+\mathcal{O}(h) .
\end{aligned}
$$

Using (5.11), (5.12), and the above identity, we get

$$
k_{\varepsilon}=\frac{1}{2} \mu^{-2} h^{2}\left(\int_{\operatorname{supp} W}\left(\int_{p}^{x} W(t) d t\right)^{2} d x+q\langle W\rangle^{2}\right)+\mathcal{O}\left(\mu^{-2} h^{3}+\mu^{-3} h^{3}\right) .
$$


In turn, the latter identity and Proposition 1 imply the claim of the theorem in the case where $p_{2}<0$.

The case of $p_{1}>0$ reduces to the above by the change of $x$ by $-x$.

Now, we pass to the case where $0 \in \operatorname{supp} W$. Since $\langle V\rangle=-\langle W\rangle$, we have

$$
\begin{aligned}
\int_{-\infty}^{\infty} & \left(\int_{-\infty}^{\frac{x}{h}} V(t) d t+\int_{-\infty}^{x} W(t) d t\right)^{2} d x \\
= & \int_{-\infty}^{-c_{0} h}\left(\int_{-\infty}^{x} W(t) d t\right)^{2} d x+\int_{-c_{0} h}^{c_{0} h}\left(\int_{-\infty}^{x h^{-1}} V(t) d t+\int_{-\infty}^{x} W(t) d t\right)^{2} d x \\
& +\int_{c_{0} h}^{\infty}\left(\langle V\rangle+\int_{-\infty}^{x} W(t) d t\right)^{2} d x \\
= & \int_{-\infty}^{0}\left(\int_{-\infty}^{x} W(t) d t\right)^{2} d x+\int_{0}^{\infty}\left(\int_{x}^{\infty} W(t) d t\right)^{2} d x+\mathcal{O}(h) .
\end{aligned}
$$

Now, (5.11) and (5.12) show that

$$
\begin{aligned}
k_{\varepsilon}= & \frac{1}{2} \mu^{-2} h^{2}\left(\int_{-\infty}^{0}\left(\int_{-\infty}^{x} W(t) d t\right)^{2} d x+\int_{0}^{\infty}\left(\int_{x}^{\infty} W(t) d t\right)^{2} d x\right) \\
& +\mathcal{O}\left(\mu^{-2} h^{3}+\mu^{-3} h^{3}\right) .
\end{aligned}
$$

Combining this with Proposition 1, we see that the theorem is valid in the case where $0 \in \operatorname{supp} W$. Theorem 1.3 is proved completely.

Proof of Theorem 1.4. In the case under consideration, formula (5.11) takes the form

$$
\tilde{\varkappa}_{\mathbf{V}, W}=\int_{-\infty}^{\infty}\left(\int_{-\infty}^{\left(x-x_{1}\right) h^{-1}} V(t) d t-\int_{-\infty}^{\left(x-x_{2}\right) h^{-1}} V(t) d t\right)^{2} d x
$$

Since

$$
\int_{-\infty}^{\infty}\left(\int_{-\infty}^{\left(x-x_{1}\right) h^{-1}} V(t) d t-\int_{-\infty}^{\left(x-x_{2}\right) h^{-1}} V(t) d t\right)^{2} d x=\int_{x_{1}}^{x_{2}}\left(\int_{-\infty}^{x} V(t) d t\right)^{2} d x+\mathcal{O}(h)
$$

we have

$$
k_{\varepsilon}=\frac{1}{2} \mu^{-2} h^{2}\left(\int_{x_{1}}^{x_{2}}\left(\int_{-\infty}^{x} V(t) d t\right)^{2} d x\right)+\mathcal{O}\left(\mu^{-2} h^{3}+\mu^{-3} h^{3}\right)
$$

by (5.14) and (5.12). Applying Proposition 1] we complete the proof.

\section{REFERENCES}

[1] L. D. Landau and E. M. Lifshits, Theoretical physics. Vol. 3. Quantum mechanics. Nonrelativistic theory, 3rd ed., Nauka, Moscow, 1974; English transl., Course of theoretical physics. Vol. 3, Quantum mechanics: nonrelativistic theory, Pergamon Press, London-Paris, 1958. MR0093319 (19:1230k)

[2] B. Simon, The bound state of weakly coupled Schrödinger operators in one and two dimensions, Ann. Phys. 97 (1976), 279-288. MR 0404846 (53:8646)

[3] M. Klaus, On the bound state of Schrödinger operators in one dimension, Ann. Phys. 108 (1977), 288-300. MR0503200 (58:20010)

[4] R. Blankenbecler, M. L. Goldberger, and B. Simon, The bound states of weakly coupled long-range one-dimensional quantum Hamiltonians, Ann. Phys. 108 (1977), 69-78. MR0456018 (56:14250)

[5] M. Klaus and B. Simon, Coupling constant thresholds in nonrelativistic quantum mechanics. I. Shortrange two-body case, Ann. Phys. 130 (1980), 251-281. MR0610664 (82h:81028a)

[6] D. I. Borisov and R. R. Gadyl'shin, On the spectrum of a periodic operator with small localized perturbation, Izv. Ross. Akad. Nauk Ser. Mat. 72 (2008), no. 4, 37-66; English transl., Izv. Math. 72 (2008), no. 4, 659-688. MR2452233 (2010b:47116)

[7] R. R. Gadyl'shin, On local perturbations of the Schrödinger operator on the axis, Teoret. Mat. Fiz. 132 (2002), no. 1, 97-104; English transl., Theoret. and Math. Phys. 132 (2002), no. 1, 976-982. MR:1956680 (2003m:34205) 
[8] I. Kh. Khusnullin, Perturbed boundary eigenvalue problem for the Schrödinger operator on an interval, Zh. Vychisl. Mat. Mat. Fiz. 50 (2010), no. 4, 679-698. (Russian) MR2761705

[9] V. P. Mikhaǐlov, Partial differential equations, Nauka, Moscow, 1976. (Russian) MR0481380 $(58: 1497)$

Bashir State Pedagogical University, Ul. Oktyabrskoi Revolyutsii 3a, Ufa 450000, Russia

E-mail address: gadylshin@yandex.ru

Bashkir State Pedagogical University, Ul. Oktyabrskoi Revolyutsir 3a, Ufa 450000, Russia

E-mail address: khusnullini@yandex.ru

Received 11/JUL/2010

Translated by B. M. BEKKER 